\title{
Dermatomyositis sine dermatitis, a rare phenotype of idiopathic inflammatory myopathy
}

\author{
Jin-Sung Park', Ji-Young Park ${ }^{2}$ \\ Departments of ${ }^{I}$ Neurology and ${ }^{2}$ Pathology, Kyungpook National University School of Medicine, Daegu, Korea
}

Dermatomyositis (DM) is characterized by progressive proximal limb weakness and typical skin manifestations. The histological findings that show perifascicular atrophy and deposition of membrane attack complex are pathognomic features of DM. Dermatomyositis is categorized into classical DM and non-classical DM, which includes amyopathic DM and DM sine dermatitis. DM sine dermatitis is seldom described because of its rarity, making the diagnosis more challenging. We report a case of DM sine dermatitis, a rare phenotype of DM.

Keywords: Dermatomyositis; Sine dermatitis; Skin manifestation; Perifascicular atrophy; Diagnostic criteria

\section{INTRODUCTION}

Dermatomyositis (DM), which is clinically characterized by progressive proximal limb weakness with typical skin manifestations, is also well known to have a close association with autoimmune disease and malignancy. Different subsets of DM have been identified, including classical DM, DM sine myositis/amyopathic DM and DM sine dermatitis [1]. Although DM sine dermatitis is described in the idiopathic inflammatory myopathy European neuromuscular center (ENMC) criteria, it is clinically under-recognized and under reported. We report our experience of a rare case of DM sine dermatitis.

\section{CASE}

A 57-year-old man presented with progressive proximal

Received: April 26, 2017, Revised: May 19, 2017

Accepted: May 25, 2017

Corresponding Author: Jin-Sung Park, Department of Neurology, Kyungpook National University School of Medicine, 130

Dongdeok-ro, Jung-gu, Daegu 41944, Korea

Tel: +82-53-200-2753, Fax: +82-53-200-2029

E-mail: neurojspark@gmail.com muscle weakness for 1 year. He had no past medical history of autoimmune disease, but was taking low dose prednisolone for 1 month prescribed by a local medical clinic for myopathy, without appropriate evaluation. Initial neurological examination showed proximal limb weakness of Medical Research Council (MRC) grade 4 in the upper limbs and MRC grade $3+$ in the lower limbs. He also had a prominent weakness in neck flexion with a MRC grade of 3 and complained of dysphagia. The deep tendon reflexes were hypoactive and he had no pathological signs. The patient had no skin manifestations like Gottron's papule, heliotrope rash or shawl signs suggestive of DM (Fig. 1A), and denied having skin lesions in the past. Laboratory tests showed normal creatine kinase (CK) level of $86 \mathrm{U} / \mathrm{L}$ (range, $<170 \mathrm{U} / \mathrm{L}$ ), but the erythrocyte sedimentation rate was mildly elevated to $57 \mathrm{~mm} / \mathrm{hr}$ (range, $<30 \mathrm{~mm} / \mathrm{hr}$ ). Autoimmune related antibodies such as anti-nuclear antibody, anti-double strand DNA, rheumatoid factor, anti-neutrophil cytoplasmic antibody, lupus anticoagulant and anti Jo-1 antibodies were negative. The electromyography showed active myopathic patterns in the biceps, extensor digitorum communis and vastus lateralis muscles. Chest and abdomen computed tomography showed no evidence of malignancy or interstitial lung disease, except for asymptomatic em-

Copyright (C) 2017 Yeungnam University College of Medicine

This is an Open Access article distributed under the terms of the Creative Commons Attribution Non-Commercial License (http://creativecommons.org/licenses/by-nc/4.0/) which permits unrestricted non-commercial use, distribution, and reproduction in any medium, provided the original work is properly cited. 
physema (Fig. 1B). The results of the endoscopy study were also unremarkable. The patient received videofluroscopy that showed no aspiration or penetration; however, a grade 3 residue was observed in the vallecular and pyriform sinus. The muscle biopsy was performed in the left biceps and the pathological findings were compatible with those of a typical DM. The histopathological findings showed prominent perifascicular atrophy with necrotic and regenerating fibers, as well as invasion of lymphocytes surrounding the blood vessels (Fig. 1C, 1D). The patient was diagnosed with DM sine dermatitis and high dose prednisolone therapy was initiated. After 6 months, there was improvement of the proximal limb weakness to MRC grade 4.

\section{DISCUSSION}

The first diagnostic criteria concerning DM, which were proposed by Bohan and Peter in 1975 [2], included clinical features such as symmetric proximal limb weakness, elevated skeletal muscle enzymes, myopathic pattern in the electromyographic study, typical muscle histology including perifascicular atrophy and typical skin lesions. Later, Dalakas and Hohlfeld proposed additional criteria that divided DM into myopathic and amyopathic patterns in 2003 [3]. These criteria commonly distinguished DM from other inflammatory myopathies with an emphasis on its typical muscle biopsy and skin manifestations. However, there are cases that are clinically and pathologically compatible with $\mathrm{DM}$, but do not meet the criteria mentioned above. In 2004, the ENMC subdivided DM into classic DM, amyopathic DM, DM sine dermatitis and non-specific myositis.

Recent studies have described amyotrophic DM as a nonclassical DM and systemic reviews have considered amyotrophic DM. A retrospective study showed a relatively high prevalence of interstitial lung disease and malignancy in $13 \%$ and $14 \%$ of amyotrophic DM patients, respectively [4]. Due to its rarity, DM sine dermatitis has not been studied, unlike DM sine myositis, which is synonymous with amyopathic DM.

According to the ENMC recommendation, diagnosis of DM sine dermatitis includes all clinical criteria of classic DM except for skin rash, elevated serum CK level, typical electromyographic findings and typical muscle biopsy findings such as perifascicular atrophy or presence of membrane attack complex depositions on blood vessels. The prevalence of DM
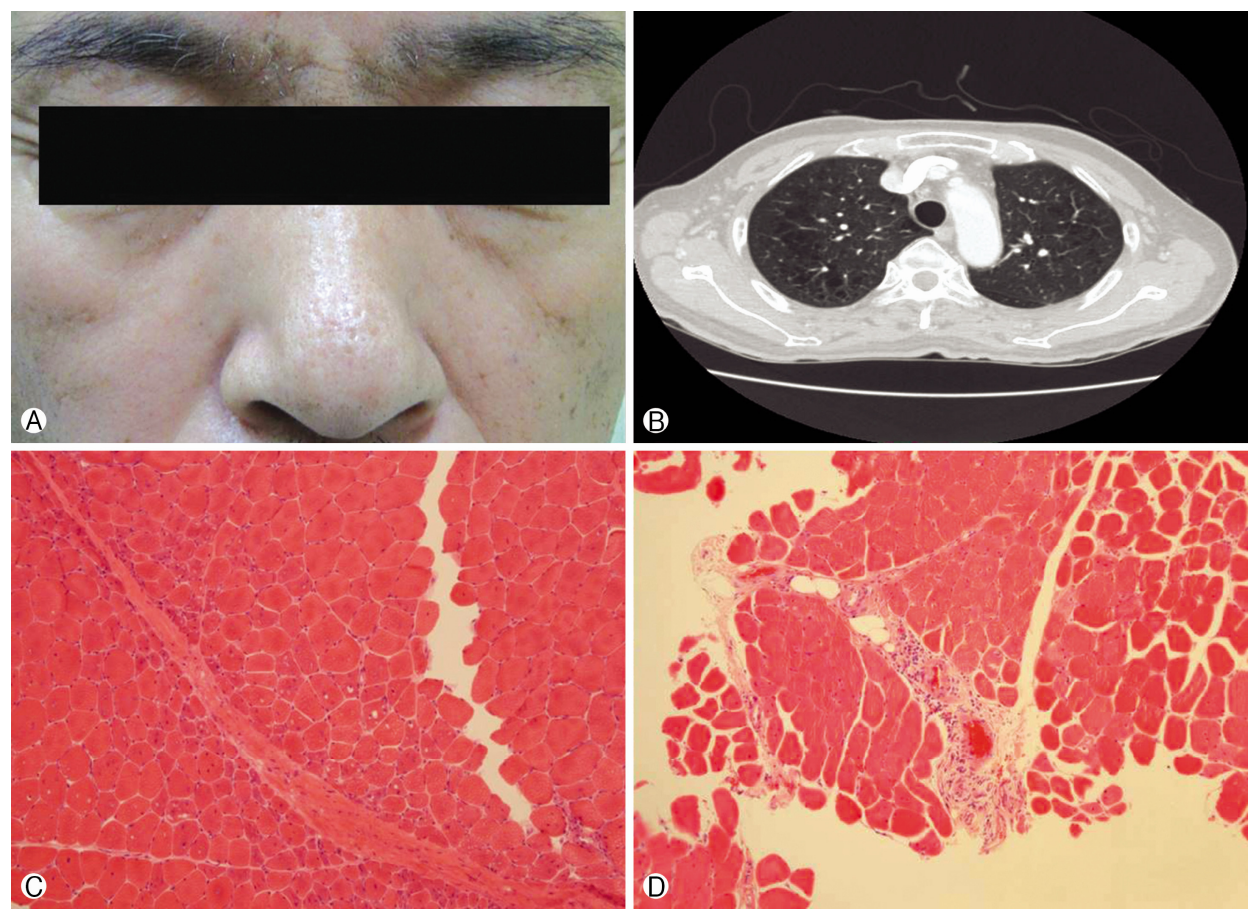

Fig 1. The patient showed no skin manifestations such as heliotrope rash (A) and only had asymptomatic emphysema upon chest computed tomography (B). The muscle biopsy of the patient illustrates a typical pattern of perifasicular atrophy $(\mathrm{C})$ and lymphocytic infiltration surrounding the blood vessel (D). 
sine dermatitis is not known, but a recent retrospective study of DM indicated a prevalence of approximately $15 \%[5]$. However these data should be considered cautiously as they showed a much lower prevalence of amyopathic DM of $2 \%$, which is inconsistent with the results of other studies that showed a prevalence rate of $10-20 \%$ [4,6]. Moreover, only one case described the clinical and pathological status of DM sine dermatitis in detail [7]. This case involved a 77-year-old male with proximal limb weakness and hyperCKemia. In accordance with our case, the patient also had dysphagia and was later found to be associated with malignancy in the kidney. The pathological findings of the previous case showed pericapillary and perivenular inflammatory infiltrates near the blood vessels. Our patient had a normal CK level, which differs from the previous case, probably because of the use of steroids before admission. Moreover, our patient showed no signs of malignancy.

Although typical skin manifestations are one of the pathognomic signs that suggest DM, we should not exclude the possibility of DM sine dermatitis. Moreover, it should be noted that confirmation via muscle biopsy is important. Finally, awareness of different subsets in DM is essential; therefore, more cases should be investigated to elucidate the existing phenotypical variability in DM.

\section{CONFLICT OF INTEREST}

The authors declare that they have no financial conflicts of interest.

\section{ORCID}

Jin-Sung Park, https://orcid.org/0000-0001-5506-9206

\section{REFERENCES}

1. Hoogendijk JE, Amato AA, Lecky BR, Choy EH, Lundberg IE, Rose MR, et al. 119th ENMC international workshop: trial design in adult idiopathic inflammatory myopathies, with the exception of inclusion body myositis, 10-12 October 2003, Naarden, The Netherlands. Neuromuscul Disord 2004;14: 337-45.

2. Bohan A, Peter JB. Polymyositis and dermatomyositis (first of two parts). N Engl J Med 1975;292:344-7.

3. Dalakas MC, Hohlfeld R. Polymyositis and dermatomyositis. Lancet 2003;362(9388):971-82.

4. Gerami P, Schope JM, McDonald L, Walling HW, Sontheimer RD. A systematic review of adult-onset clinically amyopathic dermatomyositis (dermatomyositis siné myositis): a missing link within the spectrum of the idiopathic inflammatory myopathies. J Am Acad Dermatol 2006;54:597-613.

5. Karri SB, Kannan MA, Rajashekhar L, Uppin MS, Challa S. Clinico pathological study of adult dermatomyositis: Importance of muscle histology in the diagnosis. Ann Indian Acad Neurol 2015;18:194-9.

6. Findlay AR, Goyal NA, Mozaffar T. An overview of polymyositis and dermatomyositis. Muscle Nerve 2015;51:638-56.

7. Szwebel TA, Perrot S, Kierzek G, Maisonobe T, Tigaud JM, Le Jeunne C, et al. Paraneoplasic dermatomyositis sine dermatitis associated with a tumor of the renal excretion system. J Clin Neuromuscul Dis 2008;10:35-6. 